\title{
Muscle function during takeoff and landing flight in the pigeon (Columba livia)
}

\section{Citation}

Robertson, A. M. B., and A. A. Biewener. 2012. “Muscle Function During Takeoff and Landing Flight in the Pigeon (Columba Livia)." Journal of Experimental Biology 215 (23) (December 1): 4104-4114.

\section{Published Version}

doi:10.1242/jeb.075275

\section{Permanent link}

http://nrs.harvard.edu/urn-3:HUL.InstRepos:12111359

\section{Terms of Use}

This article was downloaded from Harvard University's DASH repository, and is made available under the terms and conditions applicable to Other Posted Material, as set forth at http:// nrs.harvard.edu/urn-3:HUL.InstRepos:dash.current.terms-of-use\#LAA

\section{Share Your Story}

The Harvard community has made this article openly available.

Please share how this access benefits you. Submit a story.

Accessibility 


\title{
RESEARCH ARTICLE
}

\section{Muscle function during takeoff and landing flight in the pigeon (Columba livia)}

\author{
Angela M. Berg Robertson ${ }^{1, *}$ and Andrew A. Biewener ${ }^{2}$ \\ ${ }^{1}$ Center for Neuromotor and Biomechanics Research, University of Houston, Houston, TX 77054, USA and ${ }^{2}$ Harvard University, \\ Concord Field Station, Department of Organismic and Evolutionary Biology, 100 Old Causeway Road, Bedford, MA 01730, USA \\ *Author for correspondence (angierobertson2010@gmail.com)
}

\begin{abstract}
SUMMARY
This study explored the muscle strain and activation patterns of several key flight muscles of the pigeon (Columba livia) during takeoff and landing flight. Using electromyography (EMG) to measure muscle activation, and sonomicrometry to quantify muscle strain, we evaluated the muscle function patterns of the pectoralis, biceps, humerotriceps and scapulotriceps as pigeons flew between two perches. These recordings were analyzed in the context of three-dimensional wing kinematics. To understand the different requirements of takeoff, midflight and landing, we compared the activity and strain of these muscles among the three flight modes. The pectoralis and biceps exhibited greater fascicle strain rates during takeoff than during midflight or landing. However, the triceps muscles did not exhibit notable differences in strain among flight modes. All observed strain, activation and kinematics were consistent with hypothesized muscle functions. The biceps contracted to stabilize and flex the elbow during the downstroke. The humerotriceps contracted to extend the elbow at the upstroke-downstroke transition, followed by scapulotriceps contraction to maintain elbow extension during the downstroke. The scapulotriceps also appeared to contribute to humeral elevation. Greater muscle activation intensity was observed during takeoff, compared with mid-flight and landing, in all muscles except the scapulotriceps. The timing patterns of muscle activation and length change differed among flight modes, yet demonstrated that pigeons do not change the basic mechanical actions of key flight muscles as they shift from flight activities that demand energy production, such as takeoff and midflight, to maneuvers that require absorption of energy, such as landing. Similarly, joint kinematics were consistent among flight modes. The stereotypy of these neuromuscular and joint kinematic patterns is consistent with previously observed stereotypy of wing kinematics relative to the pigeon's body (in the local body frame) across these flight behaviors. Taken together, these observations suggest that the control of takeoff and landing flight primarily involves modulation of overall body pitch to effect changes in stroke plane angle and resulting wing aerodynamics.
\end{abstract}

Key words: bird, acceleration, maneuver.

Received 22 May 2012; Accepted 15 August 2012

\section{INTRODUCTION}

To fly effectively, a bird must successfully coordinate the muscles that move its wings. The musculature of bird wings is complex both in anatomy and in function (Dial, 1992a; Hudson et al., 1959). Because flight is a demanding form of locomotion, considerable effort has been made to understand muscle function in avian flight as a means to explore fundamental principles and limits of vertebrate muscle function. Studies of bird muscle function have revealed several biomechanical possibilities, such as: high muscle strains and strain rates (Biewener et al., 1998); exceptional work performance (Hedrick et al., 2003) and high muscle power outputs (Askew et al., 2001); control-enhancing mechanisms during flight (Tobalske and Biewener, 2008) and running (Daley et al., 2009); and remarkable abilities to modulate flight muscle power (Ellerby and Askew, 2007; Tobalske et al., 2003), leg muscle energy absorption or production (Gabaldón et al., 2004), and force sharing (Gabaldón et al., 2004; Nelson and Roberts, 2008).

The complex arrangement of the flight musculature enables birds to perform many behaviors in flight. For a given mode of flight, a particular set of muscles will be activated and change length in a coordinated manner to move the wings and achieve the desired behavior. The activation intensities of many flight muscles vary considerably among flight modes such as takeoff, landing, ascending, descending and steady flight (Dial, 1992a). Indeed, the antebrachial muscles in the pigeon (Columba livia) are unnecessary for level flapping flight, but essential for takeoff and controlled landing (Dial, 1992b). The sternobrachial portion of the pectoralis (the primary downstroke muscle) shows stronger activation during takeoff compared with other flight modes, whereas the supracoracoideus (the primary upstroke muscle) shows stronger activation during ascending flight (Dial, 1992a; Tobalske and Biewener, 2008). These observations of differential muscle activation correspond to theoretical force and power requirements of flight.

Prior to the present study, the pectoralis and supracoracoideus were the only flight muscles for which fractional length (strain) data were available. Combined with activation and force data, Biewener and colleagues described functional patterns and work output of the pectoralis in the pigeon in detail (Biewener et al., 1998). Pectoralis force peaks during the first half of the downstroke, continues after muscle activation has ceased, and falls to near zero before the upstroke begins. In the course of short flights, the pectoralis generates the most net work during the wingbeats just prior to landing, and the least net work in the initial wingbeats of takeoff (Biewener et al., 1998). Similar patterns of pectoralis strain, activation and force have more recently been observed in pigeons 
during wing-assisted incline running (Jackson et al., 2011), as well as in different-sized corvids during steep ascending flight (Jackson and Dial, 2011); and have previously been found in cockatiels, doves, budgerigars and zebra finches over a range of flight speeds (Ellerby and Askew, 2007; Hedrick et al., 2003; Tobalske and Biewener, 2008; Tobalske et al., 2003). The length and activation patterns for the pigeon supracoracoideus are similar to those of the pectoralis, though temporally shifted such that its peak force occurs at the downstroke-upstroke transition (DSUS) (Tobalske and Biewener, 2008). The supracoracoideus generates less work during takeoff and landing compared with midflight (Tobalske and Biewener, 2008).

The present study is the second in a series of investigations (see Berg and Biewener, 2010) into the free-flight performance of pigeons taking off, flying a short distance between two perches, and then landing. Here, we sought to characterize the activation and fascicle strain patterns of one extrinsic and three intrinsic flight muscles across these three flight behaviors. We collected in vivo electromyography (EMG) and sonomicrometry data from the pectoralis, as well as the biceps, humerotriceps and scapulotriceps. Our previous work on the kinematics of takeoff and landing revealed that wing kinematics differ only subtly among these flight modes (Berg and Biewener, 2010). The similarity of wing kinematics suggests that the muscle strain patterns may be similar among takeoff, midflight and landing behaviors. We nonetheless expected to observe differences in strain amplitudes among flight modes, particularly for the pectoralis, because our previous study (Berg and Biewener, 2010) showed that wingbeat amplitude is greater during takeoff than during midflight and landing. Because wing flexion at the DSUS appeared to be greatest during landing, we expected to observe greater biceps shortening and triceps lengthening during landing flight, compared with takeoff and midflight. Our kinematics results also revealed that changes in the wing stroke plane of the pigeon are largely produced by changes in body pitch (increasing $80 \mathrm{deg}$ from takeoff to landing). This observation suggested two alternative hypotheses with respect to muscle activation and strain patterns. The similar wing kinematics suggest that muscle activation and strain patterns may also be similar. Alternatively, because gravity always acts downward on the wings, yet the body and wings dramatically pitch up, different activation patterns may be necessary to maintain similar wing kinematics as the body changes its pitch orientation during flight.

Integrating in vivo muscle measurements with detailed joint kinematics enabled us to test hypotheses about the function of these muscles in flight. We expected to observe humeral depression when the pectoralis shortened; elbow flexion when the biceps shortened; elbow extension when the humerotriceps shortened; and elbow extension and humeral retraction when the scapulotriceps shortened.

The different aerodynamic requirements of takeoff, midflight and landing might also require changes in neuromuscular function. During takeoff, a bird begins flight at a low speed and accelerates forward. Most of the initial acceleration during the first wingbeat is produced by the legs (Berg and Biewener, 2010; Earls, 2000; Tobalske et al., 2004). Once aloft, aerodynamic theory (e.g. Morris and Askew, 2010a; Pennycuick, 1968; Rayner, 1979), mechanical power measurements (Askew and Ellerby, 2007; Morris and Askew, 2010b; Tobalske et al., 2003) and measurements of metabolic rate (Bundle et al., 2007; Morris et al., 2010; Tucker, 1968) suggest that flying at low speed requires greater aerodynamic power. Acceleration following takeoff to increase forward flight speed also requires a greater forward component of aerodynamic force than steady flight. Dial observed that pectoralis EMG intensities tend to
Table 1. Number of birds from which muscle data were recorded

\begin{tabular}{lcc}
\hline Muscle & Sonomicrometry & EMG \\
\hline Pectoralis & 10 & 8 \\
Biceps & 6 & 7 \\
Humerotriceps & 5 & 5 \\
Scapulotriceps & 2 & 2 \\
\hline
\end{tabular}

Five flights were analyzed for each bird.

EMG, electromyography.

be greater during takeoff from the ground and landing, compared with level flight (Dial, 1992a), reflecting the greater power and force requirements during these flight modes. We expected to observe similar patterns in the present study, but also sought to determine during which wingbeats of takeoff and landing the pectoralis was activated most strongly. Finally, because the wings move faster during takeoff and landing flight compared with midflight (Berg and Biewener, 2010), they likely produce and experience greater aerodynamic force. We therefore expected intrinsic wing muscles, such as the biceps and triceps, to show greater EMG intensity during takeoff and landing.

\section{MATERIALS AND METHODS Animals and flight arena}

All birds used in this experiment were housed at the Concord Field Station in Bedford, MA, USA, and were provided with food and water ad libitum. Twelve pigeons (Columba livia, Gmelin 1789; Table 1) were trained regularly to fly between two perches $6-8 \mathrm{~m}$ apart. The perches were made of wooden dowels $2.5 \mathrm{~cm}$ in diameter, covered with tape to provide a gripping surface for the birds, and mounted on wooden stands $\sim 1 \mathrm{~m}$ tall.

\section{Muscle function data collection}

EMG electrodes were made from twisted strands of $0.102 \mathrm{~mm}$ enamel-coated silver wire (California Fine Wire Company, Grover Beach, CA, USA), with $1 \mathrm{~mm}$ bared tips and $1-2 \mathrm{~mm}$ dipole distance. Up to three pairs of sonomicrometry crystals (2 and $1 \mathrm{~mm}$ in diameter; Sonometrics Inc., London, ON, Canada) were used for each bird. During surgery, pigeons were anesthetized to effect with isoflurane (induction, 4\%; maintenance, $0.5-2 \%$ ). Birds rested on a heating pad throughout the procedure to maintain body temperature. Surgical areas were cleansed with alcohol and feathers retracted with adhesive tape. An incision was made on the back of the bird, between the wings. Another incision was made in the skin covering the pectoralis (Fig. 1). The wires were pulled subcutaneously from the opening on the back to the opening on the ventral side of the bird, keeping the plug external to the skin. One pair of $2 \mathrm{~mm}$ crystals was implanted into the middle of the posterior pectoralis in line with the muscle's fascicle orientation, with the crystals 10-14 mm apart. EMG electrodes were implanted using a 22 gauge needle, adjacent to the fascicle where the sonomicrometry crystals were implanted.

We also investigated the function of the biceps brachii (biceps), the humerotriceps and the scapulotriceps muscles in several birds (Table 1; Fig. 1). We investigated the humeral head of the biceps, which originates along the proximal portion of the humerus and merges with the smaller, coracoidal head prior to inserting primarily on the posterior surface of the proximal end of the radius. The humerotriceps originates on the posterior surface of the shaft of the humerus and inserts on the olecranon process. The scapulotriceps originates on the lateral surface of the scapula and the posterior edge of the glenoid cavity, and inserts near the coronoid process of 


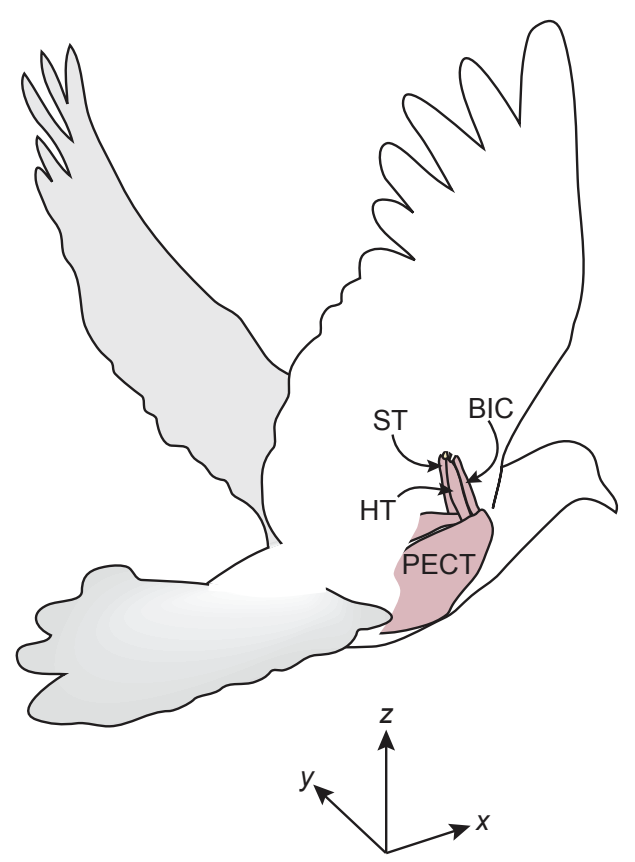

Fig. 1. Muscle anatomy of the pigeon Columba livia, and experimental coordinate system. The pectoralis (PECT) is the large ventral muscle primarily responsible for powering the downstroke. The biceps (BIC) lies anterior to the humerus, acting to flex the elbow. The short (humerotriceps, HT) and long (scapulotriceps, ST) heads of the triceps lie along the postero-ventral and postero-dorsal sides of the humerus, respectively. Both heads extend the elbow, with the biarticular scapulotriceps also serving to flex or retract the shoulder. The $x$-axis was the horizontal axis along the flight corridor, in the direction of flight; the $y$-axis was the mediolateral axis, extending horizontally from the midline of the body; the $z$-axis was the global vertical.

the ulna. More detailed visual and textual descriptions of pigeon wing anatomy can be found elsewhere (Baumel, 1979; Dial, 1992a; George and Berger, 1966).

To implant the EMG electrodes and sonomicrometry crystals into these muscles, a small incision was made over the muscles and the wires were pulled subcutaneously from the incision over the pectoralis to the incision over the more distal brachial muscles. For each of these muscles, a pair of $1 \mathrm{~mm}$ crystals was implanted in line with their fascicle orientation, as determined from previous dissections and by inspection of the anesthetized bird, with the crystals $7-10 \mathrm{~mm}$ apart. EMGs were implanted adjacent to the fascicles implanted with sonomicrometry crystals. All sonomicrometry crystals and EMGs were sutured to the muscle fascia with 4-0 silk. All skin incisions were closed with 3-0 vicryl. After surgery, birds were given flunixin megulamine $\left(1 \mathrm{mg} \mathrm{kg}^{-1}\right.$ every $12 \mathrm{~h}$ ) and recovered in a kennel with food and water ad libitum for 1-2 days prior to data collection. Before data collection, we encouraged the pigeons to fly between the perches to ensure that flight capability was not impaired by the surgery and instrumentation.

The plug on the back of the bird was connected to recording equipment via a cable. The cable had a total mass of $50 \mathrm{~g}$, but most of it rested along the floor while the bird was in flight, so the pigeon only supported an extra $\sim 10 \mathrm{~g}$ while in flight as a result of the cable. Sonomicrometry signals were received by a Triton sonomicrometrics system (Model 120-1001; Triton Technology, San Diego, CA, USA), which was connected to a computer. EMG signals were filtered $(60 \mathrm{~Hz}$ notch, $30-3000 \mathrm{~Hz}$ bandpass) and amplified at $1000 \times$ with a Grass P511 amplifier (Grass-Telefactor, West Warwick, RI, USA). The outputs from the Grass amplifier were digitized at $5000 \mathrm{~Hz}$ through a 12-bit A/D converter (Digidata 1200B, Axon Instruments, Union City, CA, USA), which was connected to the computer. Signals were recorded using AxoScope (version 8.2; Axon Instruments). During muscle data collection, birds were filmed with at least one of the high-speed digital video cameras described below.

Muscle morphology data was subsequently measured from four birds euthanized with Fatal-Plus (Vortech Pharmaceuticals Ltd, Dearborn, MI, USA). Muscle mass, mean fascicle length and pennation angle are shown in Table 2.

\section{Muscle function data analysis}

Voltage outputs from the sonomicrometry were converted to distances between the crystals using a linear conversion equation formulated with voltages corresponding to known distances for each crystal pair. Sonomicrometry distances were corrected for the epoxy forming the crystals by adding $0.82 \mathrm{~mm}$ for the distances measured with $2 \mathrm{~mm}$ crystals and $0.16 \mathrm{~mm}$ for the $1 \mathrm{~mm}$ crystals (Daley and Biewener, 2003). Sonomicrometry recordings were phase shifted to account for the $5 \mathrm{~ms}$ delay introduced by the Triton system. Muscle function data were analyzed for five flights from each of 10 birds. Sonomicrometry data were low-pass filtered at $250 \mathrm{~Hz}$, and overshoots and level shifts in the recorded signals were corrected using spline interpolation. All data filtering and analysis were performed using custom-written MatLab scripts (version 7.10, R2010a; The Mathworks Inc., Natick, MA, USA).

To estimate fascicle strain, crystal distances were normalized to the distance measured as the bird sat quietly before flight. This resting distance was determined for each flight. Strain amplitude was calculated as the difference between the maximum and minimum strains for each wingbeat. Resting lengths were consistent across flight trials.

EMG data were filtered with a $100-1000 \mathrm{~Hz}$ band-pass Butterworth filter to reduce noise and movement artifact in the recordings. Onset and offset times for each EMG burst were designated individually, by

Table2. Muscle morphology

\begin{tabular}{|c|c|c|c|c|c|c|c|c|c|c|c|c|c|c|c|c|c|}
\hline & \multirow{2}{*}{$\begin{array}{c}\text { Bird } \\
\text { mass }(\mathrm{g})\end{array}$} & \multicolumn{4}{|c|}{ Pectoralis } & \multicolumn{4}{|c|}{ Humerotriceps } & \multicolumn{4}{|c|}{ Scapulotriceps } & \multicolumn{4}{|c|}{ Biceps } \\
\hline & & Mass & $L$ & Angle & PCSA & Mass & $L$ & Angle & PCSA & Mass & $L$ & Angle & PCSA & Mass & $L$ & Angle & PCSA \\
\hline & 274 & 20.58 & 40.1 & 0 & 503.28 & 0.824 & 16.0 & 11 & 49.53 & 1.155 & 15.8 & 22 & 66.45 & 0.704 & 11.2 & 13 & 60.05 \\
\hline & 284 & 24.92 & 41.2 & 0 & 592.99 & 0.997 & 15.5 & 12 & 61.68 & 1.167 & 15.7 & 16 & 70.05 & 0.734 & 7.7 & 10 & 91.80 \\
\hline & 349 & 28.59 & 44.5 & 0 & 630.44 & 0.960 & 13.0 & 19 & 68.45 & 1.581 & 13.1 & 20 & 111.19 & 0.865 & 12.3 & 12 & 67.44 \\
\hline Mean & 303.25 & 24.37 & 43.1 & 0 & 554.99 & 0.913 & 15.5 & 13 & 56.84 & 1.349 & 15.2 & 18 & 84.07 & 0.766 & 9.6 & 15 & 77.96 \\
\hline s.d. & 33.30 & 3.34 & 2.9 & 0 & 67.39 & 0.079 & 1.9 & 4 & 9.93 & 0.220 & 1.4 & 4 & 20.52 & 0.070 & 2.5 & 7 & 16.69 \\
\hline
\end{tabular}

Measurements of muscle morphology from four birds: muscle mass (g), fascicle length ( $L$, in mm), pennation angle (deg) and physiological cross-sectional area $\left(\mathrm{PCSA}\right.$, in $\left.\mathrm{mm}^{2}\right)$. 
inspection. EMG intensity was calculated as the area under the rectified EMG trace, divided by the burst duration. To enable comparison among muscles and birds, the EMG intensities within each flight were normalized to the largest intensity observed for that flight. Unless otherwise stated, all reported EMG intensities are relative EMG intensities. Maxima and minima of sonomicrometry traces were designated individually, by inspection. For sonomicrometry and EMG traces, the upstroke-downstroke transition (USDS) was defined as the moment when the pectoralis sonomicrometry trace showed a maximum, and the DSUS as the moment when the pectoralis sonomicrometry trace showed a minimum (Fig.2).

\section{Kinematics}

Detailed kinematics of three flights from each of five birds were collected and analyzed as previously described (Berg and Biewener, 2010). Briefly, this entailed the use of high-speed digital video cameras (combinations of Photron FastCam-X 1280 PCI and Photron FastCam 1024 PCI cameras, Photron USA Inc., San Diego, CA, USA; and RedLake PCI 500 cameras, RedLake Inc., San Diego, $\mathrm{CA}, \mathrm{USA}$ ), filming at 250 frames s$^{-1}$. Birds were marked at several anatomical landmarks with non-toxic, high-contrast ink, and the points were digitized using the MatLab script DLTcalibration.m written by Ty Hedrick (Hedrick, 2008). In vivo muscle data were later collected for three of these five birds.

To enable analysis of shoulder and elbow joint kinematics relative to muscle function, additional kinematic analysis was performed for this study. Because the elbow was not marked, the position of the elbow was estimated using the positions of the shoulder and wrist, the orientation of the proximal wing plane (defined by the shoulder, wrist and rump), and approximate lengths of the humeri and radioulnae [the mean of measured distances from shoulder to elbow $(45.0 \mathrm{~mm})$ and from wrist to elbow $(65.8 \mathrm{~mm})$ from four pigeons with wings held outstretched]. Humeral anteroposterior position (protraction/retraction) and dorso-ventral position (elevation/depression) were calculated based on the position of the elbow joint, relative to the shoulder position and the body angle. Elbow joint angle was calculated as the angle between the shoulder-elbow segment and the elbow-wrist segment.

\section{Synchronization of joint angle changes and strain changes}

To relate joint and muscle function, it was necessary to synchronize the timing of the kinematic and muscle function datasets. The inertia of the distal wing causes a delay between changes in proximal muscle strain and wingtip position. Analyses of kinematic timing using more proximal measurements - such as humeral elevation/depression and wrist kinematics - were attempted, but such measurements did not exhibit a clear, consistent pattern as the wingtip did across all birds and flight modes. Thus, for each flight where muscle function was analyzed, the timing of the kinematic USDS was determined (the moment when the wingtip was dorsal and most medial), relative to the timing of maximum pectoralis strain. This delay was found to be $7.9 \pm 0.7 \mathrm{~ms}$, averaged across all birds and wingbeats. For analysis, the kinematic data were shifted by this delay in order to synchronize the shoulder and elbow temporally with the sonomicrometry and EMG data. Unless otherwise indicated, all further references to kinematic data refer to the temporally synchronized kinematic data.

Wingbeats for both muscle and kinematic data were numbered using a method described previously (Berg and Biewener, 2010). The first USDS of takeoff was designated the beginning of wingbeat 1 , which continued until the following USDS. Takeoff wingbeats were numbered sequentially as wingbeats 1,2 , 3, etc. (Fig. 3). During

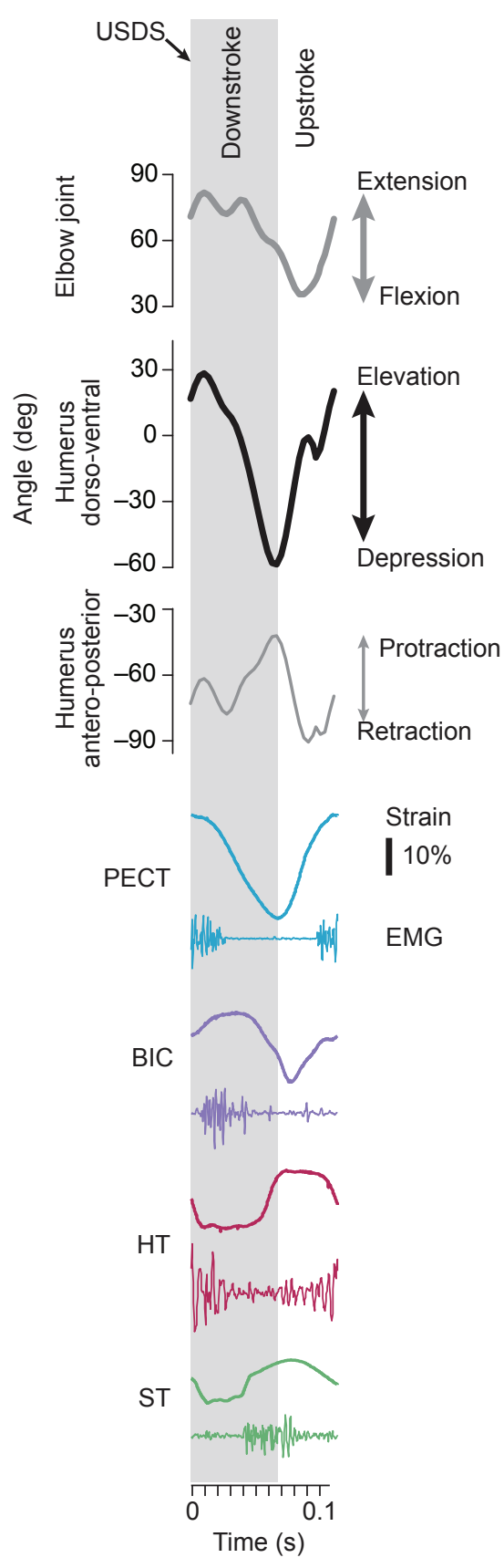

Fig. 2. Example traces of kinematics, sonomicrometry and electromyography (EMG) recordings from a single wingbeat (wingbeat 4 of Fig. 3). The gray vertical band indicates downstroke, as determined by the maximum and minimum strains of the pectoralis. The elbow joint was most flexed at the downstroke/upstroke transition (DSUS), and was extended for most of downstroke. The humerus was protracted at DSUS, and was retracted from mid-upstroke to mid-downstroke. As expected, the humerus was most elevated at the upstroke/downstroke transition (USDS) and most depressed at DSUS. Scale bar indicates $10 \%$ strain for all muscle strain traces. Descriptions of muscle function traces are provided in Results.

landing, the birds did not always make contact with the perch ('footdown') during the same phase of the wingbeat. High-speed video was used to determine the timing of foot-down for both kinematic and muscle data collection. To standardize the wingbeat numbers relative to the moment when the feet touched the perch, the wingbeat that included foot-down was considered 'wingbeat 0 ', generally. More 


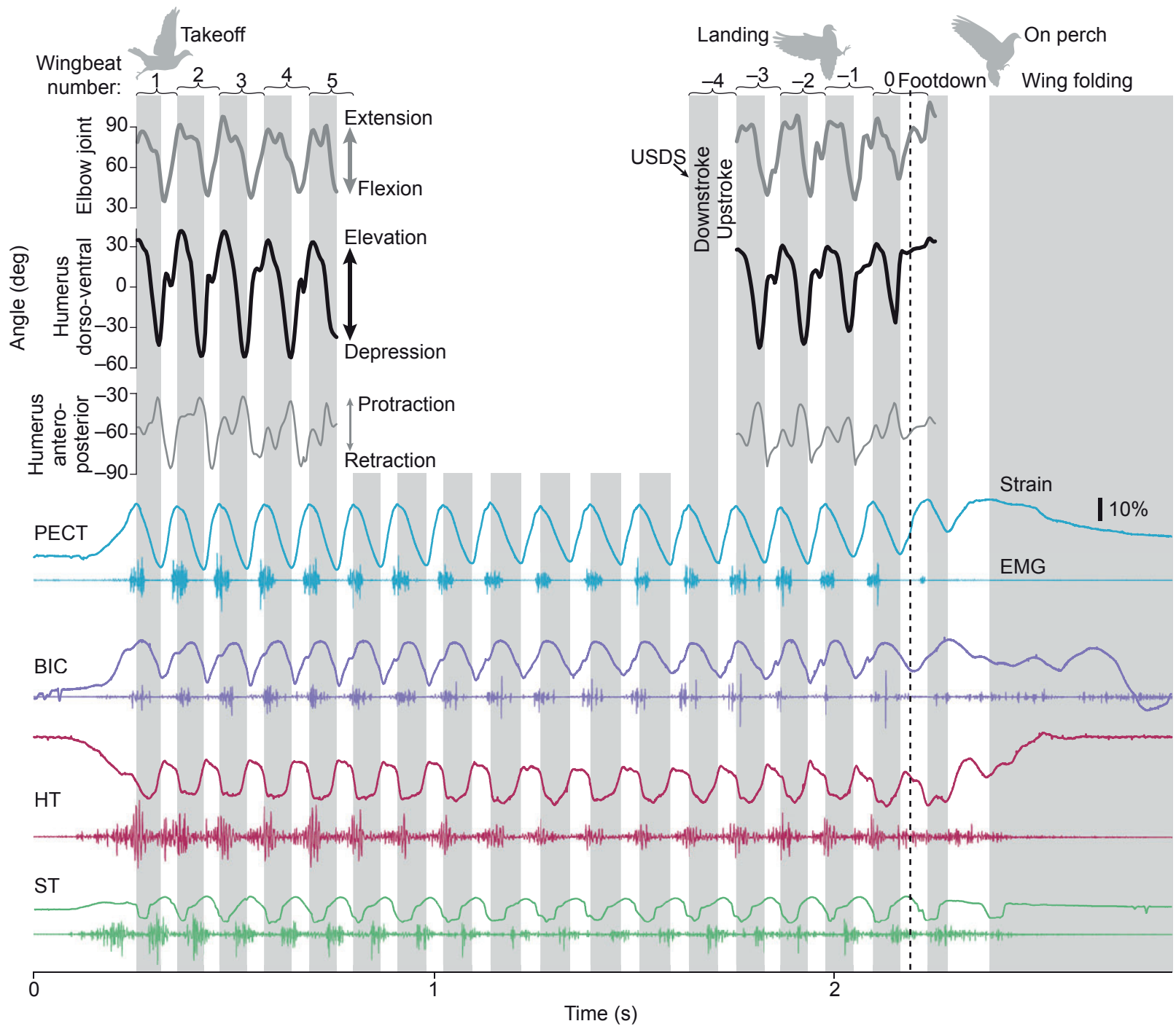

Fig. 3. Sample traces of kinematics, sonomicrometry and EMG recordings. See Fig. 2 for traces of a single wingbeat and Fig. 6 for overall averages. Gray vertical bands indicate downstroke; USDS are therefore the left edge of the gray bands. Kinematic traces and traces of pectoralis, humerotriceps and scapulotriceps data are from flights of bird 9. Traces of biceps data are from bird 1. (Not all muscles could be recorded at one time from any single bird; in this figure, therefore, biceps and kinematic data have been scaled to the pectoralis strain pattern shown in order to reflect the typical timing of kinematic and biceps strain patterns.) Silhouettes indicate example body positions near the USDS for the flight modes. Wingbeat numbers are determined as described in Materials and methods. Scale bar indicates $10 \%$ strain for all muscle strain traces. Birds showed consistent general patterns of muscle strain and activation among each other. Descriptions of muscle function traces are provided in Results.

specifically, 'wingbeat 0 ' ended either after foot-down or less than $20 \mathrm{~ms}$ before foot-down (i.e. the USDS that occurred after foot-down or less than $20 \mathrm{~ms}$ before foot-down defined the end of wingbeat 0 ). Wingbeats prior to wingbeat 0 were numbered sequentially as wingbeats $-1,-2$, etc. (Fig. 3).

Because the biceps, humerotriceps, and scapulotriceps exhibited strains that did not follow simple sawtooth patterns, strain rates were calculated for each wingbeat phase for all muscles. Each wingbeat was divided into several phases of particular interest: USDS; early-, mid- and late-downstroke (DS); DSUS; early-, mid- and late-upstroke (US) (Table 3). The early, mid and late phases each comprised onethird of the respective half-stroke. The transition phases, USDS and DSUS, included the final sixth of the prior stroke and first sixth of the next stroke, overlapping with the early- and late-DS and US phases.

Table 3. Timing of wingbeat phases and events relative to percentage of wingbeat cycle based on kinematics

\begin{tabular}{lcccccccc}
\hline & USDS & Early-DS & Mid-DS & Late-DS & DSUS & Early-US & Mid-US & Late-US \\
\hline Wingbeat phase & -6 to 11 & 0 to 22 & 22 to 43 & 43 to 64 & 59 to 67 & 64 to 76 & 76 to 88 & 88 to 100 \\
Wingbeat event & 0 & & 32 & & 64 & 82 & 82 \\
\hline
\end{tabular}

All data are $\%$ of wingbeat cycle (means of grand means).

Negative values indicate the previous wingbeat cycle. 
Table 4. Timing of kinematic and motor events

\begin{tabular}{lcccc}
\hline & Pectoralis & Biceps & Humerotriceps & Scapulotriceps \\
\hline Peak length & 0 (=USDS) & 21 & 57 & 72 \\
Fastest shortening phase & Mid-DS & Late-DS & USDS & Late-US \\
Minimum length & 64 (=DSUS) & 69 & 12 & 18 \\
Fastest lengthening phase & Early to mid-US & Mid-US & Late-DS to DSUS & Late-DS \\
Activation begins & -7 (prior wingbeat) & 0 & -34 (prior wingbeat) & 40 \\
Activation ends & 35 & 43 & 36 & 85 \\
\hline
\end{tabular}

Numbers indicate the percentage of the wingbeat cycle, where $0 \%$ is the USDS.

All data are means of grand means. See also Figs 4, 5.

For each wingbeat phase, strain change was calculated for each muscle. Unless otherwise noted, all fascicle strain rates reported are for the phase with the maximum shortening rate (Table 4).

\section{Statistical analysis}

Data were averaged within each bird for each wingbeat. Repeatedmeasures (rm) ANOVA tests and post hoc tests were performed on these means in IBM SPSS Statistics (version 20.0.0; IBM Corp., Armonk, NY, USA). We applied the sequential Bonferroni correction to determine the significance of rm ANOVA and post hoc tests (Rice, 1989). For post hoc comparisons among wingbeats, which numbered 55, the Bonferroni correction required that there be a $P$-value less than 0.000909 for any wingbeat comparison to be ruled significant. Bird mean data for each wingbeat were averaged to generate grand means for each wingbeat, which are illustrated in the figures. Some grand means were further averaged over the wingbeats of full flights or takeoff or landing. Because averaging the data three times may render the standard errors and statistical tests unreliable, these standard errors are generally not reported and claims of statistical significance are not made based on these averages. Otherwise, values are reported as means \pm s.e.m.

\section{RESULTS}

\section{Muscle strain and activation patterns}

Birds showed consistent general patterns of muscle strain and activation among individuals. Example traces are illustrated in Figs 2, 3; and strains and timings are summarized in Figs 4-6. Details of the timing of kinematic and motor events are reported in Tables 3 and 4. The pectoralis strain exhibited a simple, sawtooth-like pattern, with the shortening phase $64 \%$ of wingbeat duration. The pectoralis was active from late-US until mid-DS, reflecting its role in reversing the wingstroke at the USDS and producing downstroke lift. Previous work has demonstrated that the pectoralis produces force after the EMG burst has ended (34 ms, 19\% of wingstroke cycle) (Biewener et al., 1998), and we assume a similar EMG-force delay for the other muscles described here.

The biceps shortened during the second half of downstroke and was shortest slightly after the DSUS, when the elbow was most flexed. The biceps then lengthened during most of upstroke as the elbow extended. The biceps was active from the USDS through the first two-thirds of downstroke, suggesting a role in stabilizing the elbow while the wing produces aerodynamic lift during the downstroke. Only two birds exhibited a second burst of biceps activity like that described previously (Dial et al., 1991). The biceps strain remained near its peak for a longer period of time than the pectoralis, resulting in a relatively isometric contraction during the USDS or first half of downstroke.

The humerotriceps shortened most rapidly at the USDS to extend the elbow and wing, then remained at a relatively uniform length until late-DS, when it lengthened again and remained at a longer uniform length until late-US. The humerotriceps was active from early-US to mid-DS of the next wingbeat, indicating that it actively shortened during the USDS to extend the elbow. The scapulotriceps also functioned at two relatively uniform lengths, shortening between mid-US and USDS to extend the elbow, and lengthening at late-DS. The scapulotriceps was active from midDS until mid-US, indicating that it contracts nearly isometrically when it is at its longer length.

The muscles studied here differed from each other in their strain and activation patterns (Figs 4-6). The pectoralis consistently averaged the greatest fascicle strain amplitudes across wingbeats (range 0.239-0.341). The humerotriceps and scapulotriceps averaged lower strain amplitudes throughout the flights, with overlapping ranges (0.071-0.103 and $0.097-0.118$, respectively). The biceps contracted with strain amplitudes that were intermediate to the pectoralis and the triceps (humerotriceps and scapulotriceps) muscles (range 0.157-0.255). The greater strains observed in the biceps compared with its antagonists, the triceps, may be the result of the biceps having shorter fascicles than the triceps (in relation to each muscle's moment arm at the elbow joint).

The pectoralis exhibited its greatest shortening rate during midDS; the biceps during late-DS; the humerotriceps during USDS; and the scapulotriceps during late-US (Table4). Comparisons and illustrations of fascicle strain rates are based on these maximum shortening rates. The biceps contracted with the greatest maximum shortening rates (grand means range $3.18-8.29 L^{-1}$, where $L$ is length), followed by the pectoralis $\left(3.62-5.71 \mathrm{~L} \mathrm{~s}^{-1}\right)$, the scapulotriceps (2.91-3.93 $\left.\mathrm{L} \mathrm{s}^{-1}\right)$ and the humerotriceps, which had the lowest fascicle strain rates $\left(1.37-1.98 \mathrm{~L} \mathrm{~s}^{-1}\right)$.

Variation of muscle strain and activation with flight mode Muscle strain amplitude varied significantly among wingbeats for all four muscles (rm ANOVA, $F \geq 2.785, P \leq 0.008$; Fig. 4A). The pectoralis showed significant differences in mean strain amplitude between several wingbeats, most notably: takeoff wingbeat 2, which showed the highest values $(0.341)$; midflight, which showed lower values than takeoff ( 0.306 ; $t$-tests, $P<0.0012$, except versus wingbeat 1$)$; and the final landing wingbeat, which showed values lower than landing wingbeat -1 ( $t$-test, $P=0.0004)$. For other muscles, post hoc comparisons among wingbeats were not significant with the Bonferroni correction, which required a very low $P$-value for significance $(P<0.0009)$ because of the large number of comparisons (55). The pectoralis shortened during an average of $66 \%$ of wingbeat duration for all wingbeats except wingbeats $1(63 \%)$ and $0(53 \%)$, which varied significantly from most other wingbeats ( $\mathrm{rm}$ ANOVA, $F=22.403, P<0.0005$ for wingbeat 1 versus $2-5$, midflight, and $-2 ; P<0.0009$ for wingbeat 0 versus all others). 

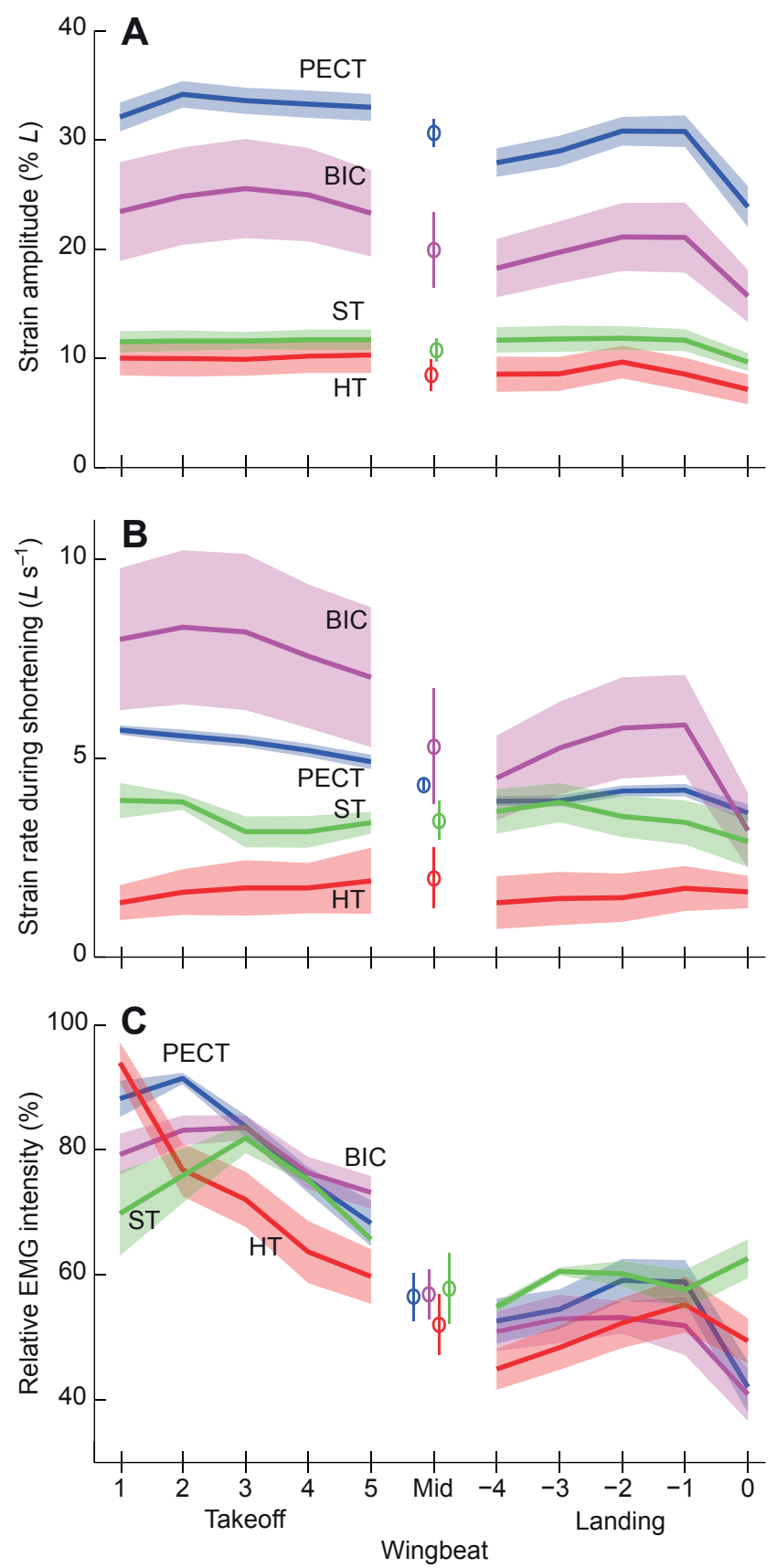

Fig. 4. Muscle fascicle strain amplitude (A), strain rate (B) and relative EMG intensity (normalized to the maximum signal recorded from the muscle for each flight, C) across flight modes. Graphs show the mean \pm s.e.m. (shading) across individuals for each variable. The pectoralis contracted with a greater strain amplitude and strain rate during takeoff than during midflight or landing. The biceps exhibited its highest strain rates at the beginning of takeoff, and the lowest strain amplitude and strain rate during midflight and landing wingbeats. The triceps muscles showed relatively consistent strain amplitudes and strain rates throughout the flight, and with respect to each other. All muscles showed the greatest EMG intensity within the first three wingbeats of takeoff and exhibited lower EMG intensity during landing.

Pectoralis fascicle strain rate varied significantly among wingbeats (rm ANOVA, $F=58.436, P<0.0005$; Fig. 4B), and most post hoc wingbeat comparisons were also significant ( $t$-tests, $P<0.004$; except wingbeat 1 versus 2 and $3, P \geq 0.23$; and among some landing wingbeats, $P \geq 0.21$ ). Wingbeat 1 showed the greatest strain rate $\left(5.71 \mathrm{~L} \mathrm{~s}^{-1}\right)$. During landing, pectoralis strain amplitude and strain rate increased from wingbeats -4 to -2 and then dropped from wingbeat -1 to 0 . The biceps showed a similar pattern of strain rates, which varied significantly among wingbeats ( $\mathrm{rm}$ ANOVA, $F=5.728$, $P<0.0005)$. Although post hoc $t$-test wingbeat comparisons were nonsignificant with the Bonferroni correction, the $t$-test of pooled takeoff wingbeats versus pooled landing wingbeats yielded $P=0.031$. The humerotriceps and scapulotriceps showed consistent values of strain rate throughout the flights (rm ANOVA, $F \leq 1.355, P \geq 0.320$ ).

Muscle activation intensity varied significantly among wingbeats for all muscles except the scapulotriceps (scapulotriceps: rm ANOVA, $F=2.004, P=0.144$; other muscles: rm ANOVA, $F \geq 19.146$, $P<0.0005$; Fig. 4 C). Activation intensity of the pectoralis, biceps and humerotriceps was greatest during the takeoff. For these muscles, activation intensity did not vary from midflight through landing ( $t$-tests, $P \geq 0.0028$ ).

The timing of muscle activation did not change dramatically across the three phases of takeoff, midflight and landing (Figs 2, 3). No muscle was observed to be active during shortening in one flight mode but active during lengthening in another flight mode. Nonetheless, some trends in the timing of activation and muscle length change were apparent (Fig. 5). During the final landing wingbeat (0), the downstroke duration shortened by $20 \%$, causing most motor pattern timing features to shift to an earlier point in the wingbeat cycle. The onset times for the pectoralis, humerotriceps and scapulotriceps varied significantly among wingbeats ( $\mathrm{rm}$ ANOVA, $F \geq 9.662, P \leq 0.0005$ ); and the offset times for the pectoralis and biceps varied significantly among wingbeats (rm ANOVA, $F \geq 3.190, P \leq 0.002)$. Post hoc comparisons showed that pectoralis onset times relative to USDS of takeoff wingbeats 1 and 2 were significantly earlier than in subsequent takeoff, midflight and landing wingbeats ( $t$-tests, $P \leq 0.00103$ ); the pectoralis offset time for wingbeat 0 was significantly earlier than most other wingbeats ( $t$-tests, $P \leq 0.0007$, except versus wingbeats $1,-3$ and -4 ).

\section{Kinematics and muscle strain}

Patterns of humeral position and elbow joint angles varied little across wingbeats and flight modes (Fig. 6B). Kinematics of wingbeats 1 and 0 occasionally differed slightly from the mean values of the grand means (within flight modes). Muscle strain corresponded with humerus and elbow position in expected and informative patterns (Figs 6, 7). As expected, the humerus depressed when the pectoralis shortened. When the biceps shortened during the second half of downstroke, the elbow flexed -49.0 deg (mean of grand means). The elbow extended slightly during the first half of the downstroke (mean of grand means: $4.9 \mathrm{deg}$ change), when the biceps was nearly isometric. Most of the elbow extension occurred between early-US and USDS, when the humerotriceps and scapulotriceps shortened. Interestingly, when the humerus was elevated, the scapulotriceps was shortened. Some paths tracing these parameters through the wingbeat cycle are fairly linear, suggesting simple relationships between muscle strain and wing kinematics. These paths include the pectoralis strain versus dorso-ventral humerus position; and the biceps, humerotriceps and scapulotriceps strains versus elbow joint angle.

\section{DISCUSSION}

In this study, we sought to characterize the strain and activation patterns of several flight muscles to determine how their functions differ among takeoff, midflight and landing behaviors. This also represents the first study to measure strains in key intrinsic wing muscles of a bird in flight. 


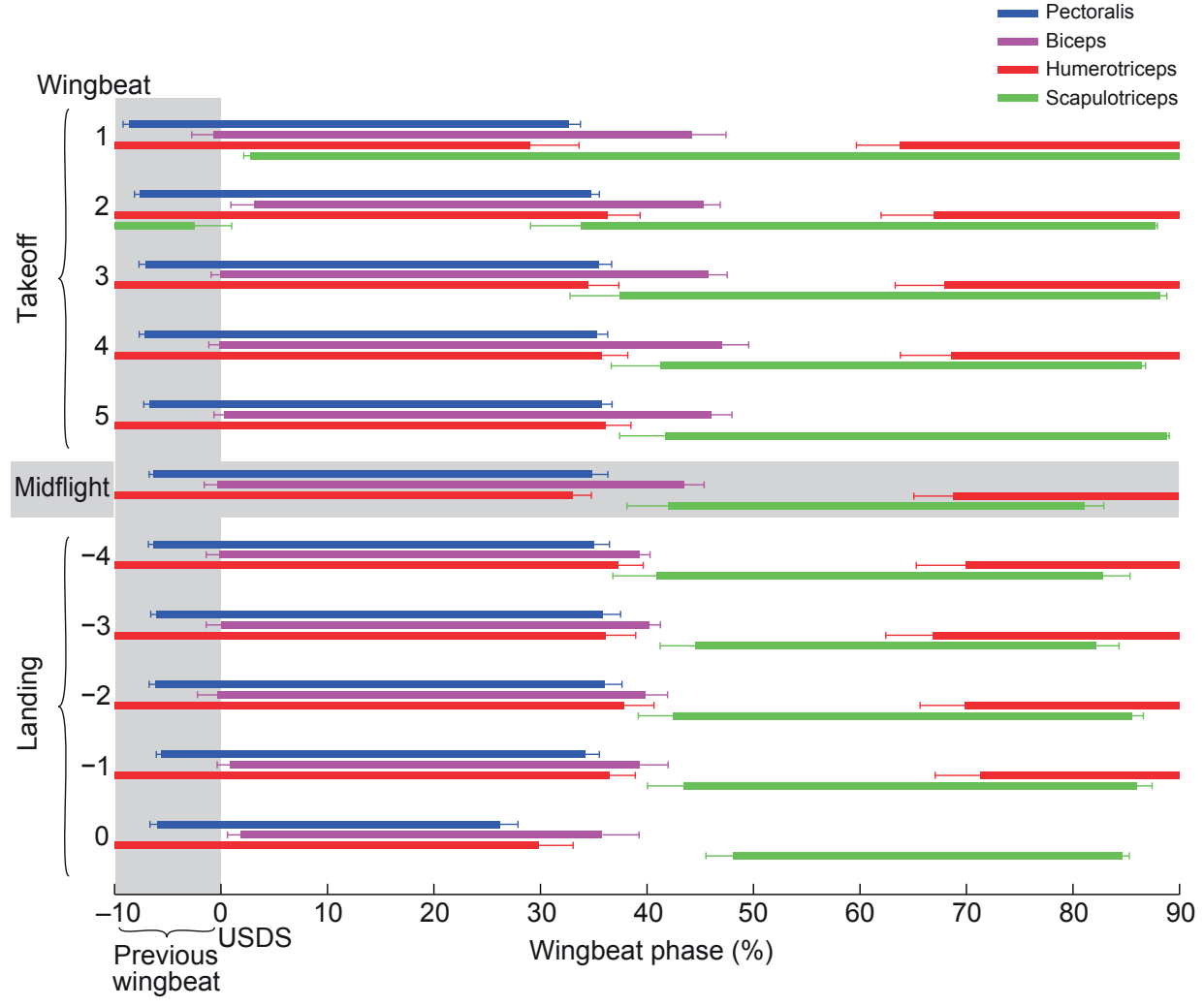

Fig. 5. Summary of timing of motor events in the wingbeat cycle. This figure summarizes the temporal patterns of muscle activation observed over all birds, for each wingbeat. The wingbeat phase axis extends from $-10 \%$ to $90 \%$ to simplify illustration of the timing of pectoralis activation. Wingbeat numbers correspond to the four adjacent colored bars. Bars represent activation of each muscle (see key). The right and left edges of the bars indicate the mean onset and offset timings of the muscle, respectively. Whiskers on the bars indicate s.e.m. in onset/offset timings. The maximum pectoralis length (USDS) defines the beginning of the wingbeat $(0 \%)$. The most notable change across wingbeats occurs in the final landing wingbeat $(0)$, where the timings of several motor pattern events are shifted to an earlier phase in the wingbeat. This corresponds to the shorter downstroke observed during this wingbeat (Berg and Biewener, 2010). Other observable trends include the earlier onset of scapulotriceps activation during takeoff.

\section{Muscle strain and activation patterns}

Consistent with previous observations in pigeons (Biewener et al., 1998; Dial, 1992a; Jackson et al., 2011; Tobalske and Biewener, 2008) and other avian species (Ellerby and Askew, 2007; Jackson et al., 2011; Tobalske et al., 2003), the pectoralis showed an asymmetric saw-tooth strain pattern and was active from late-US until mid-DS. Asymmetric saw-tooth strain patterns have been shown to be an efficient mechanism for increasing muscle power output (Askew and Marsh, 1997; Holt and Askew, 2012), and the possibility exists for animals to adjust the symmetry of muscle length trajectories in order to modulate power. Interestingly, we found that during the first and final wingbeats of short flights, the pectoralis strain pattern was more symmetrical - not less so - despite the expectation that these wingbeats would have the greatest power requirements. This observation suggests that in short flights, pigeons do not take advantage of this theoretical mechanism to enhance power output during the slowest wingbeats. Although muscle force was not measured, we used the timing of muscle activation as an approximation for the timing of active force production, recognizing that there is likely a substantial time delay for muscle force relaxation following the end of muscle activation. Previous work has shown that the pectoralis produces force well after activation has ceased (34 ms, 19\% of wingstroke cycle) (Biewener et al., 1998). Because all wing muscles must operate at a similar cycle frequency (that of the wingbeat), it is plausible that they share similar contractile kinetics, and a similar EMG-force delay is suggested for the other muscles in the following discussion.

Not surprisingly, we found that the biceps exhibited its minimal fascicle strain near the DSUS when the elbow was flexed. The biceps was active and nearly isometric during the first two-thirds of the downstroke, suggesting that it acts to stabilize the elbow during downstroke (Figs 2, 6). These patterns are consistent with the biceps muscle function previously proposed (Dial, 1992a). An EMG-force delay similar to that of the pectoralis would suggest that the biceps also produces force while shortening and flexing the elbow during the last third of the downstroke. These observations imply that the biceps produces force during both lengthening and shortening, resulting in negative work during the first portion of downstroke and positive work during the later portion of downstroke. The negative work of the biceps implies that the muscle also acts to absorb some of the kinetic energy of the distal wing as it rapidly extends in the first half of downstroke.

We found that the scapulotriceps was active while it was lengthened, from late-DS until late-US, suggesting that the scapulotriceps acts to stabilize the elbow before and after the DSUS. The scapulotriceps lengthens slightly while active, indicating that it may produce some negative work as the elbow flexes and the humerus depresses. Because of the delay in force relaxation after the end of its activation, the scapulotriceps likely produces force throughout its shortening phase as it extends the wing at the end of upstroke, thereby producing positive work. The humerotriceps contracted with low shortening strain during most of downstroke, lengthened during the DSUS as the elbow flexed, and then remained at a long length for most of upstroke, consistent with elbow flexion during this phase of the wingbeat cycle. The humerotriceps became active after mid-US, contracting when it shortened to extend the wing at the USDS. The humerotriceps then contracted nearly isometrically until mid-DS, suggesting that it may actively maintain wing extension during the DS, as well as stabilize the elbow concurrently with the biceps for the first half of the downstroke. These strain and activation patterns imply that the humerotriceps produces positive work to extend the elbow at the USDS. The activation timings observed for both triceps muscles are similar to patterns previously observed for pigeons (Dial, 1992a) and starlings (Dial et al., 1991), and corroborate previously proposed triceps muscle functions (Dial, 1992a). 
A
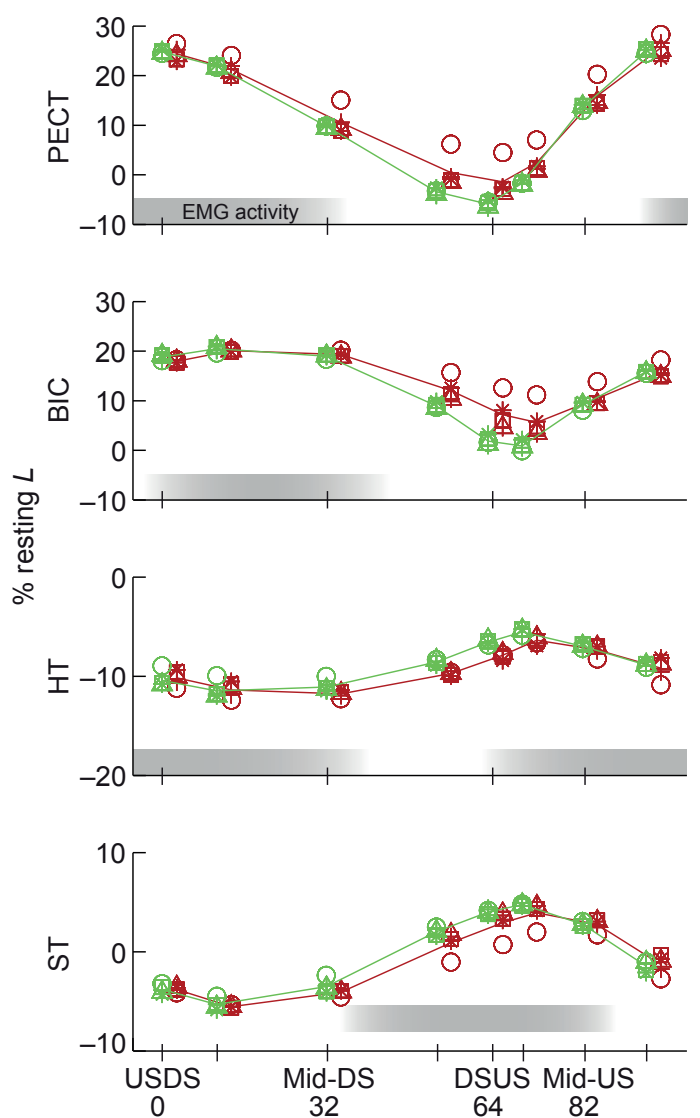

B
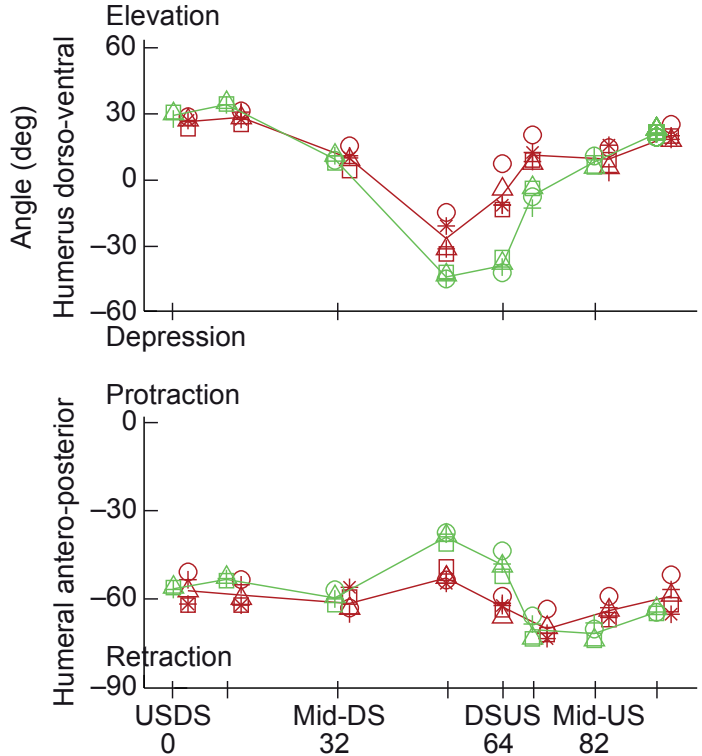

Fig. 6. Mean muscle fascicle strain (A) and kinematic angle (B) across wingbeat phases. The $x$-axis shows the percentage of the wingbeat cycle and timing of wingbeat events (see also Table 3). Tick marks indicate wingbeat phases (USDS, earlyDS, etc.). Green lines illustrate mean values for takeoff wingbeats; red lines illustrate landing wingbeats. Takeoff and landing data are offset - takeoff left of landing - for clarity. Symbols illustrate mean values for specific wingbeats. The extensive overlap among the wingbeats illustrates the similarity of wingbeat strain and kinematic patterns across wingbeats. The final landing wingbeat (0), indicated by red circles, showed observable differences in strain and kinematic values from other wingbeats. Gray bands on graphs of strain $(A)$ indicate when the muscle is typically active (mean of grand means; see also Fig. 7).
Although both heads of the triceps appear to contribute to elbow extension near the USDS, the difference between the humerotriceps and scapulotriceps in their relative timing of activation suggests that they have additional, differentiating functions: the scapulotriceps to stabilize the wing at the DSUS, and the humerotriceps to stabilize the elbow at the USDS through mid-DS. Anatomical differences between the humerotriceps and scapulotriceps may explain the differences in their strain amplitudes. The fascicle lengths are similar between the humerotriceps and scapulotriceps (Table2), but the scapulotriceps is biarticular, crossing the elbow and the shoulder and originating from the scapula, whereas the humerotriceps crosses only the elbow, having its origin from the proximal humerus. The scapulotriceps therefore lengthens not only when the elbow flexes but also when the humerus depresses. Thus, because the humerus simultaneously depresses as the elbow flexes, the scapulotriceps is lengthened more than the humerotriceps.

\section{Muscle function patterns across flight modes}

Because wing kinematics have been shown to vary primarily as a result of changes in body orientation from takeoff to landing (Berg and Biewener, 2010), we expected intrinsic muscle strain amplitudes to vary little across these flight modes. This proved to be the case, though the biceps tended to show greater strain amplitude during takeoff than during midflight and landing. We expected the pectoralis strain amplitude to reflect the differences in stroke amplitude observed in our previous work (Berg and Biewener, 2010), and this proved to be the case. The pectoralis showed significantly greater strain amplitude during takeoff than during midflight and landing. Pectoralis strain amplitude increased at the beginning of landing and then decreased in the final wingbeats of landing. Biceps strain was greater than that observed for the humerotriceps and scapulotriceps, which may be due to shorter fascicle lengths in the biceps. Additionally, the scapulotriceps is biarticular, and some of the strain observed may be the result of strains linked to shoulder movement (described below).

The onset and offset timing of muscle activation relative to shortening and lengthening did not change substantially among flight modes. This suggests that muscles that produce positive work during midflight also produce positive work during takeoff and landing, and vice versa. Two trends across flight mode were observed with respect to timing. During the final landing wingbeat (0), many timing features of the motor pattern were shifted to an earlier phase in the wingbeat (Fig. 5). This reflects the shorter downstroke amplitude that pigeons employ during the final wingbeat when landing on a perch (Berg and Biewener, 2010).

We tested two alternative hypotheses with respect to muscle activation intensity. The apparent similarity of wing kinematics across flight modes suggested that muscle activation patterns may be uniform during flight. Alternatively, the dramatic pitch rotation of the body from takeoff to landing may require altered activation intensities to maintain stereotypic wing kinematics with respect to gravity. Consistent with the latter hypothesis, the activation intensities of the pectoralis, biceps and humerotriceps were significantly different across flight modes. It remains unclear whether the relationship between muscle activation intensity and the pitch rotation is correlative or causal. Forthcoming research into the aerodynamics of takeoff and landing in the pigeon or indepth analysis of center of mass mechanics (e.g. Ros et al., 2011) 

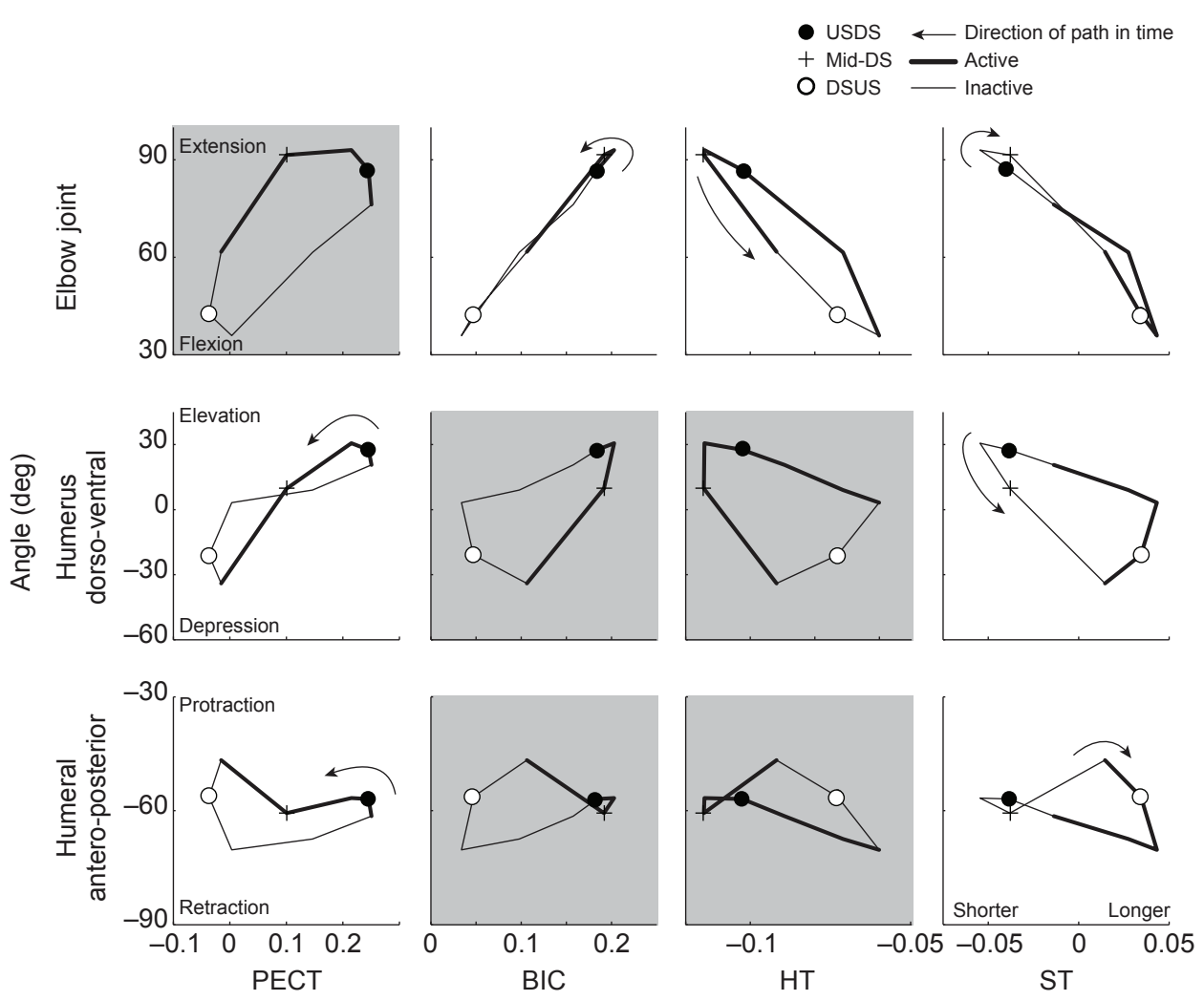

Strain (\% resting $L$ )
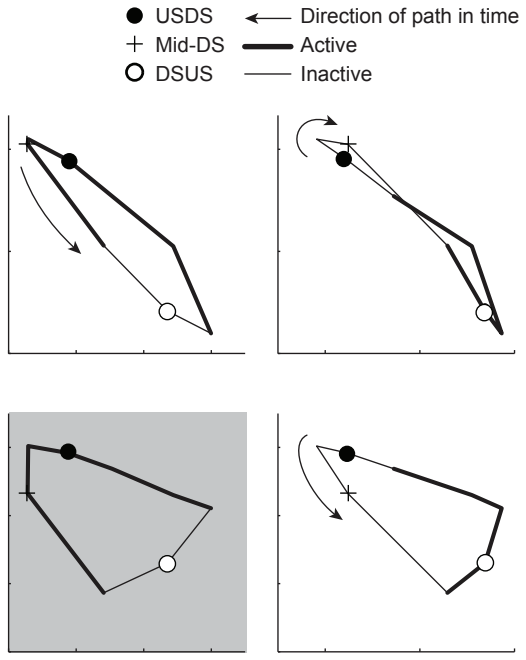

Fig. 7. Mean humerus positions (with respect to the shoulder) and elbow joint angles, versus muscle fascicle strain, with EMG timings overlaid. (See also Fig. 6.) Loops trace the paths of the mean of the grand means. Filled circles indicate the USDS; open circles indicate the DSUS; and arrows indicate the temporal direction of the paths. Thickened bands on the paths indicate the activation period for the muscle indicated on the $x$-axis (see also Fig. 5). As expected, when the pectoralis is shorter, the humerus is depressed. When the biceps shortens, the elbow flexes; and likewise, when the humerotriceps and scapulotriceps shorten, the elbow extends. Notably, when the scapulotriceps is shorter, the humerus is also elevated. Corroborating other evidence discussed in the text, this observation suggests that the scapulotriceps contributes to the elevation of the humerus. The anteroposterior position of the humerus may have been influenced by the pectoralis and scapulotriceps, but none of the muscles investigated here showed a definitive pattern with respect to humeral protraction and retraction. Relationships that are definitely correlative and not causative (based on anatomy) are shown with a gray background. may answer this question. However, the small shift in net aerodynamic force orientation required to produce the observed changes in body pitch from take-off to midflight (maximum moment arm estimated to be $\sim 1.4 \mathrm{~mm}$ ) and then from midflight to landing $(\sim 1.7 \mathrm{~mm})$ (Berg and Biewener, 2010) will make the underlying mechanism challenging to demonstrate. The present data suggest that the control of aerodynamic force production responds to varying gravitational forces experienced by the wings. The pectoralis showed greater activation intensities during takeoff relative to midflight and landing. This corresponds with the greater force production of the pectoralis during takeoff flight (Biewener et al., 1998; Dial and Biewener, 1993). The greater activation intensity, fascicle shortening strain and force output of the pectoralis during takeoff downstrokes together reflect the increased aerodynamic requirements for forward acceleration and support of body weight at the low speeds of takeoff flight.

\section{Kinematics and muscle function}

This study showed that simultaneous changes in kinematics and muscle strain followed expected patterns, but also revealed noteworthy details in these parameters (Figs 6, 7). As expected, humeral depression was observed when the pectoralis shortened. But the present results also suggest that the action of the scapulotriceps explains the slight humeral elevation observed just prior to DSUS: the scapulotriceps is anatomically well positioned to contribute to humeral elevation, but moreover, it is active during DSUS and is shortened when the pectoralis is elevated. Some caution is necessary in drawing such a conclusion, as the data here show correlation, not necessarily causation. This observation could potentially be explained by simultaneous elbow extension by the scapulotriceps and humeral elevation by the supracoracoideus, which was not one of the muscles investigated here.
Elbow flexion occurred when the biceps shortened, and elbow extension occurred when the humerotriceps and scapulotriceps shortened, indicative of the role of the biceps as the primary elbow flexor and the roles of the humerotriceps and scapulotriceps as elbow extensors. Because the pectoralis does not insert distal to the elbow, its relationship with elbow flexion is definitely correlative and not causative. Similarly, because the biceps (humeral head) and humerotriceps do not originate proximal to the shoulder, their relationships with humeral movement are also definitely correlative and not causative. The antero-posterior position of the humerus may have been influenced by the pectoralis and scapulotriceps, but none of the muscles investigated here showed a definitive pattern with respect to humeral protraction and retraction. Nonetheless, based on the anatomy and activation timings of these muscles, it is likely that many of the observed strain changes contributed to the observed kinematic changes of the joints these muscles cross.

\section{CONCLUSION}

Muscle strain and activation intensity of the pectoralis, biceps and triceps generally showed greater values during takeoff, compared with slow level and landing flight modes. Yet, qualitative withinwingbeat patterns varied little among wingbeats of different flight modes. This stereotypy of muscle activation and strain patterns is also reflected in the consistent joint kinematics from takeoff to landing. Timing patterns of strain and activation were also consistent throughout these short flights. These similarities in motor and kinematic patterns among flight modes may provide a mechanism that simplifies the control of wing motion during nonsteady flight. Despite the complex musculoskeletal anatomy and high aerodynamic demands of avian flight, behaviors as different as takeoff and landing may be controlled by simple changes in the magnitude of flight muscle contractile parameters such as 
strain, strain rate and muscle activation intensity, facilitated by aerodynamic changes that result from rotating the wing stroke plane and body.

\section{ACKNOWLEDGEMENTS}

We would like to thank our colleagues at the Concord Field Station for their help in data collection. We particularly thank Alison Hsiang and Jonathan Barr for their assistance as undergraduates helping to carry out the experiments. We thank Pedro Ramirez for caring for the birds, and the other staff members at the Concord Field Station for providing help with logistics. We are also grateful for the constructive, insightful comments of two anonymous reviewers.

\section{FUNDING}

This work was supported by the National Science Foundation [grant IOS-0744056 to A.A.B.], together with funds through the Harvard University Organismic and Evolutionary Biology graduate program. A.M.B.R. was supported in part by a grant from the Joe W. King Orthopedic Institute [UH-98166] to Daniel O'Connor, via the Health and Human Performance Department of the University of Houston.

\section{REFERENCES}

Askew, G. N. and Ellerby, D. J. (2007). The mechanical power requirements of avian flight. Biol. Lett. 3, 445-448.

Askew, G. N. and Marsh, R. L. (1997). The effects of length trajectory on the mechanical power output of mouse skeletal muscles. J. Exp. Biol. 200, 3119-3131.

Askew, G. N., Marsh, R. L. and Ellington, C. P. (2001). The mechanical power output of the flight muscles of blue-breasted quail (Coturnix chinensis) during takeoff. J. Exp. Biol. 204, 3601-3619.

Baumel, J. J. (1979). Nomina Anatomica Avium. New York: Academic Press.

Berg, A. M. and Biewener, A. A. (2010). Wing and body kinematics of takeoff and landing flight in the pigeon (Columba livia). J. Exp. Biol. 213, 1651-1658.

Biewener, A. A., Corning, W. R. and Tobalske, B. W. (1998). In vivo pectoralis muscle force-length behavior during level flight in pigeons (Columba livia). J. Exp. Biol. 201, 3293-3307.

Bundle, M. W., Hansen, K. S. and Dial, K. P. (2007). Does the metabolic rate-flight speed relationship vary among geometrically similar birds of different mass? J. Exp. Biol. 210, 1075-1083.

Daley, M. A. and Biewener, A. A. (2003). Muscle force-length dynamics during level versus incline locomotion: a comparison of in vivo performance of two guinea fowl ankle extensors. J. Exp. Biol. 206, 2941-2958.

Daley, M. A., Voloshina, A. and Biewener, A. A. (2009). The role of intrinsic muscle mechanics in the neuromuscular control of stable running in the guinea fowl. J. Physiol. 587, 2693-2707.

Dial, K. P. (1992a). Activity patterns of the wing muscles of the pigeon (Columba livia) during different modes of flight. J. Exp. Zool. 262, 357-373.

Dial, K. P. (1992b). Avian forelimb muscles and nonsteady flight: can birds fly without using the muscles in their wings? Auk 109, 874-885.

Dial, K. P. and Biewener, A. A. (1993). Pectoralis muscle force and power output during different modes of flight in pigeons (Columba livia). J. Exp. Biol. 176, 31-54.
Dial, K. P., Goslow, G. E. and Jenkins, F. A. (1991). The functional-anatomy of the shoulder in the European starling (Sturnus vulgaris). J. Morphol. 207, 327-344.

Earls, K. D. (2000). Kinematics and mechanics of ground take-off in the starling Sturnis vulgaris and the quail Coturnix coturnix. J. Exp. Biol. 203, 725-739.

Ellerby, D. J. and Askew, G. N. (2007). Modulation of pectoralis muscle function in budgerigars Melopsitaccus undulatus and zebra finches Taeniopygia guttata in response to changing flight speed. J. Exp. Biol. 210, 3789-3797.

Gabaldón, A. M., Nelson, F. E. and Roberts, T. J. (2004). Mechanical function of two ankle extensors in wild turkeys: shifts from energy production to energy absorption during incline versus decline running. J. Exp. Biol. 207, 2277-2288.

George, J. C. and Berger, A. J. (1966). Avian Myology. New York: Academic Press.

Hedrick, T. L. (2008). Software techniques for two- and three-dimensional kinematic measurements of biological and biomimetic systems. Bioinspir. Biomim. 3, 034001.

Hedrick, T. L., Tobalske, B. W. and Biewener, A. A. (2003). How cockatiels (Nymphicus hollandicus) modulate pectoralis power output across flight speeds. J. Exp. Biol. 206, 1363-1378.

Holt, N. C. and Askew, G. N. (2012). The effects of asymmetric length trajectories on the initial mechanical efficiency of mouse soleus muscles. J. Exp. Biol. 215, 324-330.

Hudson, G. E., Lanzillotti, P. J. and Edwards, G. D. (1959). Muscles of the pelvic limb in galliform birds. American Midland Naturalist 61, 1-67.

Jackson, B. E. and Dial, K. P. (2011). Scaling of mechanical power output during burst escape flight in the Corvidae. J. Exp. Biol. 214, 452-461.

Jackson, B. E., Tobalske, B. W. and Dial, K. P. (2011). The broad range of contractile behavior of the avian pectoralis: functional and evolutionary implications. J. Exp. Biol. 214, 2354-2361.

Morris, C. R. and Askew, G. N. (2010a). Comparison between mechanical power requirements of flight estimated using an aerodynamic model and in vitro muscle performance in the cockatiel (Nymphicus hollandicus). J. Exp. Biol. 213, 2781-2787.

Morris, C. R. and Askew, G. N. (2010b). The mechanical power output of the pectoralis muscle of cockatiel (Nymphicus hollandicus): the in vivo muscle length trajectory and activity patterns and their implications for power modulation. J. Exp. Biol. 213, 2770-2780

Morris, C. R., Nelson, F. E. and Askew, G. N. (2010). The metabolic power requirements of flight and estimations of flight muscle efficiency in the cockatiel (Nymphicus hollandicus). J. Exp. Biol. 213, 2788-2796.

Nelson, F. E. and Roberts, T. J. (2008). Task-dependent force sharing between muscle synergists during locomotion in turkeys. J. Exp. Biol. 211, 1211-1220.

Pennycuick, C. J. (1968). Power requirements for horizontal flight in the pigeon (Columba livia). J. Exp. Biol. 49, 527-555.

Rayner, J. M. V. (1979). A new approach to animal flight mechanics. J. Exp. Biol. 80 17-54.

Rice, W. R. (1989). Analyzing tables of statistical tests. Evolution 43, 223-225.

Ros, I. G., Bassman, L. C., Badger, M. A., Pierson, A. N. and Biewener, A. A. (2011). Pigeons steer like helicopters and generate down- and upstroke lift during low speed turns. Proc. Natl. Acad. Sci. USA 108, 19990-19995.

Tobalske, B. W. and Biewener, A. A. (2008). Contractile properties of the pigeon supracoracoideus during different modes of flight. J. Exp. Biol. 211, 170-179.

Tobalske, B. W., Hedrick, T. L., Dial, K. P. and Biewener, A. A. (2003). Comparative power curves in bird flight. Nature 421, 363-366.

Tobalske, B. W., Altshuler, D. L. and Powers, D. R. (2004). Take-off mechanics in hummingbirds (Trochilidae). J. Exp. Biol. 207, 1345-1352.

Tucker, V. A. (1968). Respiratory exchange and evaporative water loss in the flying budgerigar. J. Exp. Biol. 48, 67-87. 